\title{
Shermo: A general code for calculating molecular thermochemistry properties
}

\author{
Tian $\mathrm{Lu},{ }^{[\mathrm{a}], *}$ Qinxue Chen, ${ }^{[\mathrm{a}]}$ \\ [a] Beijing Kein Research Center for Natural Sciences, Beijing 100022, P. R. China \\ (http://www.keinsci.com) \\ * Correspondence author. E-mail: $\underline{\text { sobereva@sina.com }}$
}

\section{ORCiD}

Tian Lu: 0000-0002-1822-1229

Qinxue Chen: 0000-0003-0155-2387

\begin{abstract}
Calculation of molecular thermodynamic quantities is one of the most frequently involved task in daily quantum chemistry studies. In this article, we present a general, stand-alone, powerful and flexible code named Shermo for calculating various common thermochemistry data. This code is compatible with Gaussian, ORCA, GAMESS-US and NWChem and has many unique advantages: the output information is very easy to comprehend; thermodynamic quantities can be fully decomposed to contributions of various sources; temperature and pressure can be conveniently scanned; two quasi-rigid-rotor harmonic oscillator (quasi-RRHO) models are supported to properly deal with low frequencies; different frequency scale factors can be simultaneously specified for calculating different thermodynamic quantities; conformation weighted thermodynamic data can be directly evaluated; the code can be easily run and embedded into shell script. We hope the Shermo program will bring great convenience to quantum chemists. This code can be freely obtained at http://sobereva.com/soft/shermo.
\end{abstract}

Keywords: Shermo, thermochemistry, thermodynamic, conformation, free energy, molecular vibration, frequency scale factor, RRHO, harmonic approximation, Gaussian, ORCA 


\section{Introduction}

Calculation of molecular thermodynamic quantities, such as enthalpy, Gibbs free energy and heat capacity, is the most frequently involved problem in daily quantum chemistry research. Due to its importance, almost all popular quantum chemistry packages have their own code to compute thermodynamic data. However, these codes often have various shortcomings and limitations, for example: (1) No better model beyond harmonic approximation for low frequency modes is supported (2) Frequency scale factors for different purposes cannot be separately specified at the same time (3) Data is inconvenient to recalculate at other temperatures and pressures (4) Temperature and pressure cannot be easily scanned (5) Contribution to thermodynamic data from various vibrational modes cannot be individually printed (6) Conformation weighting cannot be directly taken into account (7) Output information of some programs is complicated and thus difficult for beginners to understand. Since these problems have caused great inconveniences to researchers in the field of quantum chemistry, we believe it is quite necessary to develop a powerful, flexible and easy-to-use code for calculating molecular thermodynamic quantities to address aforementioned issues. Due to this consideration, a code named Shermo was recently developed by us and will be introduced in this article. Briefly speaking, Shermo is a stand-alone and versatile molecular thermodynamic calculation code based on output file of frequency analysis task of mainstream quantum chemistry programs, including Gaussian, ${ }^{[1]}$ ORCA, ${ }^{[2]}$ GAMESS-US ${ }^{[3]}$ and NWChem. ${ }^{[4]}$ Since Shermo makes molecular thermochemistry analysis unprecedentedly convenient, we believe this code will become a new important member in many quantum chemists' toolbox.

In Section 2, we will describe the model employed by Shermo for calculating thermodynamic data, in Section 3 various features of Shermo will be introduced. A few application examples of using Shermo to study practical chemical problems will be given in Section 4. Finally, in Section 5 we summarize this article.

\section{Calculation model}

Shermo calculates molecular thermodynamic data mostly based on rigid-rotor harmonic oscillator model under ideal gas assumption, which is also employed in thermochemistry analysis of almost all quantum chemistry codes. This model has been very detailedly documented in manual of Shermo and also introduced in many books, ${ }^{[5,6]}$ therefore full introduction of this model will not be presented here, only key points will be mentioned.

Shermo is able to calculate all common molecular thermodynamic data, including internal energy $(U)$, enthalpy $(H)$, Gibbs free energy $(G)$, entropy $(S)$, heat capacity of constant volume $\left(C_{\mathrm{V}}\right)$ and constant pressure $\left(C_{\mathrm{P}}\right)$, as well as molecular partition function $(q)$. All of them can be decomposed as contributions from translation, rotation, vibration and electronic excitation by ignoring their couplings, which usually is a reasonable assumption. In Shermo, these contributions can be printed separately.

The rotation contribution is dependent of molecular principal moments of inertia $(I)$ and rotational symmetry number $(\sigma)$. The $I$ is computed based on atomic masses and current geometry, while $\sigma$ is determined by point group of current molecule. Obviously, correct identification of point group is crucial. In Shermo, point group is automatically detected by our modified version of SYVA 
code, ${ }^{[7]}$ which is fairly robust and fast.

The description of the vibration part has key influence on calculation accuracy of thermodynamic quantities. Usually, the frequencies involved in the calculation are obtained based on harmonic approximation, which is the simplest model of representing molecular vibrations. In this case, the vibrational contribution to various thermodynamic data can be calculated as follows

$$
\begin{aligned}
& U(0)_{\mathrm{vib}}=\mathrm{ZPE}=\frac{1}{2} \sum_{i} h v_{i} \\
& U_{0 \rightarrow T}^{\mathrm{vib}} \equiv U(T)_{\mathrm{vib}}-U(0)_{\mathrm{vib}}=R T \sum_{i}\left(\frac{h v_{i}}{k T}\right) \frac{e^{-h v_{i} /(k T)}}{1-e^{-h v_{i} /(k T)}} \\
& C_{\mathrm{v}, \mathrm{vib}}(T)=R \sum_{i}\left(\frac{h v_{i}}{k T}\right)^{2} \frac{e^{-h v_{i} /(k T)}}{\left[1-e^{-h v_{i} /(k T)}\right]^{2}} \\
& S_{\mathrm{vib}}(T)=R \sum_{i}\left\{\frac{h v_{i}}{k T} \frac{e^{-h v_{i} /(k T)}}{1-e^{-h v_{i} /(k T)}}-\ln \left[1-e^{-h v_{i} /(k T)}\right]\right\}
\end{aligned}
$$

where $R$ is ideal gas constant, $k$ is Boltzmann constant, $T$ stands for temperature, $v_{i}$ denotes vibrational frequency of mode $i$. As can be clearly seen, all of them can be viewed as summation of contribution of various vibrational modes. In order to make analysis of the nature of the quantities feasible, Shermo allows printing individual contribution coming from different vibrational modes.

It is well known that neglect of anharmonic effect and inaccurate representation of potential energy surface must lead to significant error in thermodynamic quantities. Fortunately, this kind of error is relatively systematical and thus can be largely eliminated by applying pre-fitted scale factor for harmonic frequencies prior to calculation of various thermochemistry data. Frequency scale factors $(\lambda)$ corresponding to various calculation levels have been fitted in many literatures, some of them provided $\lambda$ for zero-point energy $\left(\lambda_{\mathrm{ZPE}}\right),{ }^{[8-13]}$ while some other ones also presented $\lambda$ for entropy $\left(\lambda_{\mathrm{S}}\right)$ and heating contribution to internal energy or enthalpy $\left(\lambda_{\text {heat }}\right){ }^{[14-17]}$ Usually both $\lambda_{\mathrm{S}}$ and $\lambda_{\text {heat }}$ are close to 1.0 , while $\lambda_{\mathrm{ZPE}}$ deviates from 1.0 notably, therefore applying a global frequency scale factor in all thermodynamic data calculations is evidently inappropriate. In Shermo, the $\lambda_{\mathrm{ZPE}}$, $\lambda_{\mathrm{s}}, \lambda_{\text {heat }}$ and $\lambda$ for heat capacity can be simultaneously specified and properly considered in the calculation of various thermodynamic data.

The harmonic approximation treatment for vibrations, which is often referred to as rigid-rotor harmonic oscillator (RRHO) model, breaks down for very low frequency modes. This point can be easily understood from below equations, which show contribution to entropy and heating contribution to internal energy due to vibration mode $i$.

$$
\begin{aligned}
& S^{i}(T)=R\left\{\frac{h v_{i}}{k T} \frac{1}{e^{h v_{i} /(k T)}-1}-\ln \left[1-e^{-h v_{i} /(k T)}\right]\right\} \\
& U_{0 \rightarrow T}^{i}(T)=\left(\frac{h v_{i}}{k T}\right) \frac{R T}{e^{h v_{i} /(k T)}-1}
\end{aligned}
$$

Clearly, as the $v_{i}$ approaches zero, the above two quantities diverge because their denominators tend to vanish. In order to make calculation result for a system containing low frequencies more reasonable, Shermo supports two popular methods to tackle this issue and they are usually referred to as quasi-RRHO (QRRHO). The first QRRHO treatment is the one originally suggested by Truhlar and coworkers, see refs. $[18,19]$ for example. This method artificially raises all frequencies that 
lower than $100 \mathrm{~cm}^{-1}$ to $100 \mathrm{~cm}^{-1}$, the cutoff can be customized in Shermo. Recently, in an article of studying aqueous $\mathrm{p} K_{\mathrm{a}}$ prediction, ${ }^{[20]}$ it was shown that for small molecules, the accuracy of calculation result is insensitive to the choice of the cutoff, while for molecular complexes, the original cutoff $\left(100 \mathrm{~cm}^{-1}\right)$ performs best.

Another QRRHO treatment supported by Shermo was proposed by Grimme, ${ }^{[21]}$ and it only applies to entropy. Essentially, this method performs interpolation between the vibrational entropy calculated under harmonic approximation $\left(S^{\mathrm{RRHO}}\right)$ and the one calculated based on free-rotor model $\left(S^{\mathrm{FR}}\right)$, the latter can be expressed as

$$
S_{\mathrm{FR}}=R\left(\frac{1}{2}+\ln \sqrt{\frac{8 \pi^{3} \mu^{\prime} k T}{h^{2}}}\right)
$$

with

$$
\mu^{\prime}=\frac{\mu B_{\mathrm{av}}}{\mu+B_{\mathrm{av}}} \quad \mu=\frac{h}{8 \pi^{2} v}
$$

where $\mu$ is moment of inertia of a free-rotor with same frequency as the vibrational mode, $\mu^{\prime}$ corresponds to effective moment of inertia. The $B_{\text {av }}$ denotes average molecular moment of inertia and was empirically chosen to be $10^{-44} \mathrm{~kg} \cdot \mathrm{m}^{2}$. According to our experience, the result of the two aforementioned QRRHO models do not differ with each other notably, while both of them may improve accuracy of free energy computed by RRHO model considerably for highly flexible systems, which are usually accompanied by large number of low frequencies. As indicated in ref. [21], the error of RRHO on large organic systems containing 300 400 atoms can easily reach as high as $3 \sim 4 \mathrm{kcal} / \mathrm{mol}$ at standard condition. Therefore, we always suggest using one of the QRRHO models in Shermo instead of RRHO. Relatively speaking, we prefer to employ the Grimme's method since it has more rigorous physical background than the way of artificially raising low frequencies.

Electronic excitation also contributes to thermodynamic quantities and can be considered in the calculation as long as user specifies energy levels in Shermo; for example, its contribution to entropy is expressed as

$$
S_{\text {ele }}=R \ln \left(\sum_{i} g_{i} e^{-\varepsilon_{i} /(k T)}\right)+R \frac{\sum_{i} g_{i}\left[\varepsilon_{i} /(k T)\right] e^{-\varepsilon_{i} /(k T)}}{\sum_{i} g_{i} e^{-\varepsilon_{i} /(k T)}}
$$

where $\varepsilon_{i}$ is electronic energy level $i$ with respect to ground state, $g_{i}$ is degeneracy of level $i$. Note that contribution of electronic excitation is not considered in thermodynamic analysis code of almost all quantum chemistry programs, since additional calculation is needed to evaluate excitation energies, and energy separation between lowest excited state and ground state is usually as large as several $\mathrm{eV}$, in this case contribution due to electron excitations is fully negligible. However, extremely low-lying excited states, for example, the ones caused by spin-orbit coupling splitting of ground state, ${ }^{[5]}$ should be taken into account in thermodynamic data calculation. When ground state is degenerate solely due to electron spin, Eq. 5 reduces to

$$
S_{\text {ele }}=R \ln g_{0}
$$

where $g_{0}$ in this context corresponds to spin multiplicity. Since Shermo automatically identifies spin multiplicity from output file of quantum chemistry program, this kind of electron contribution to 
entropy is always automatically considered.

Flexible molecules usually have many thermal accessible conformations, while molecular complexes usually have many thermal accessible configurations. Appropriate consideration of these conformations or configurations is crucial for obtaining reliable and meaningful thermodynamic data. The conformation (or configuration) weighted thermodynamic data can be obtained via the following way

$$
U^{\mathrm{w}}=\sum_{i}^{\text {conf }} p_{i} U_{i} \quad C_{V}^{\mathrm{w}}=\sum_{i}^{\text {conf }} p_{i} C_{V, i} \quad S^{\mathrm{w}}=S^{\mathrm{conf}}+\sum_{i}^{\text {conf }} p_{i} S_{i}
$$

where $p$ stands for Boltzmann weight, which can be determined based on relative Gibbs free energies between the conformers. ${ }^{[22]}$ The quantities with subscript $i$ denote the data calculated for

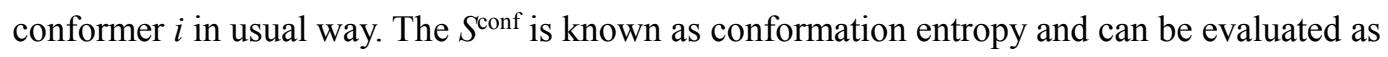

$$
S^{\mathrm{conf}}=-R \sum_{i}^{\mathrm{conf}} p_{i} \ln p_{i}
$$

The conformation weighted Gibbs free energy can be evaluated as $G^{\mathrm{w}}=H^{\mathrm{w}}-T S^{\mathrm{w}}$. In Shermo, thermochemistry quantities and Boltzmann weights for all conformers as well as conformation weighted thermodynamic data can be directly outputted, users just need to provide a list containing path of output files of frequency analysis task for all thermal accessible conformers.

\section{Characteristics of Shermo}

\subsection{Basic characteristics}

Shermo is a code written in Fortran, its executable file of Windows and Linux platforms as well as a detailed manual can be freely downloaded at http://sobereva.com/soft/shermo. Source code can also be freely obtained upon request from us.

Shermo can be run very easily without any installation step or preparation of running environment. After booting up Shermo, running parameters will be loaded from "settings.ini" file, and user will be asked to input path of the input file, then molecular information and various thermodynamic quantities will be printed on screen.

Shermo can also run purely via command line, for example:

$$
\text { Shermo /nico/maki.out -P } 5 \text {-sclZPE } 0.96 \text {-ilowfreq } 2>11 \text {.txt }
$$

This command invokes Shermo to deal with "/nico/maki.out", the output information is redirected to "ll.txt", the pressure is set to be $5 \mathrm{~atm}$, the frequency scale factor for ZPE is set to be 0.96 , and Grimme's QRRHO treatment for entropy is employed. All other parameters that not explicitly specified will employ the values defined in the "settings.ini" file. Clearly, Shermo can be conveniently embedded into shell script to automatically process large amount of files.

\subsection{Input file}

Output file of harmonic frequency analysis task of Gaussian, ORCA, GAMESS-US and NWChem can be directly used as input file of Shermo. Geometry optimization can also be simultaneously conducted, for example, output file of "opt freq" task of Gaussian and ORCA is also acceptable. Shermo loads all necessary information that needed by calculating thermochemistry 
quantities from input file, including electronic energy, spin multiplicity, elements and coordinates of all atoms, and harmonic frequencies.

For flexibility, Shermo also defined a private format with "shm" as suffix. This file contains everything that needed by Shermo calculation, thus it can also be employed as input file. The shm format is concise and clear, thus one can easily manually create or modify it. The shm file for a typical molecule HCN is illustrated in Figure 1. The shm file can also be directly generated by Shermo after loading output file of quantum chemistry program.

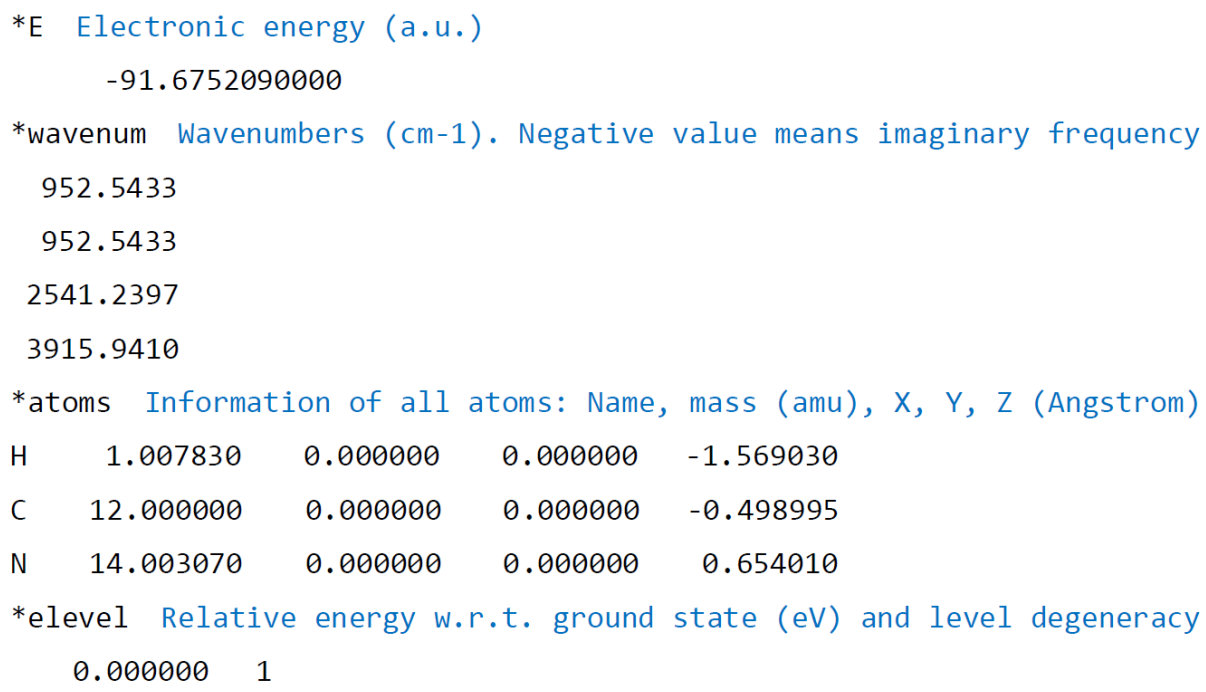

Figure 1 shm file of HCN molecule. Blue texts are comments

Due to the generality of the shm format, users of other quantum chemistry programs may also make use of Shermo to compute thermodynamic data, what they need to do is extracting data from output file of the programs manually or through their own code and then write the data into a file with the shm format.

To compute conformation weighted data, a list file should be prepared and used as input file, each of its lines records path of a frequency analysis output file of a conformer. For example:

D: \yohane \conf1.out;233.463126

D: \yohane \conf2.out; 233.476224

D: \yohane \conf3.out;233.458291

The values after semicolon are electronic energies in a.u., which usually should be calculated at a level better than frequency analysis, since electronic energy dominates internal energy, enthalpy and Gibbs free energy and meantime it is highly sensitive to calculation level. If the electronic energies are not explicitly provided, they will be directly loaded from the output files of frequency analysis task.

\subsection{Running parameters}

Running parameters of Shermo are loaded from the "settings.ini" file or directly specified via arguments in command line. The parameters are summarized in Table 1, detailed introduction can be found in manual. 
Table 1 Running parameters in Shermo program.

\begin{tabular}{|c|l|}
\hline E & Electronic energy. If not explicitly specified, it will be loaded from input file \\
\hline prtvib & If printing contribution of each vibrational mode \\
\hline $\mathrm{T}$ & Temperature used for calculation \\
\hline $\mathrm{P}$ & Pressure used for calculation \\
\hline sclZPE & Frequency scale factor for ZPE \\
\hline sclheat & Frequency scale factor for heating contribution to $U$, namely $U(T)-U(0)$ \\
\hline sclS & Frequency scale factor for entropy \\
\hline sclCV & Frequency scale factor for heat capacity \\
\hline ilowfreq & Model for dealing with low frequencies \\
\hline ravib & Cutoff of raising low frequencies \\
\hline outshm & If exporting .shm file after loading output file of quantum chemistry program \\
\hline defmass & Type of default atomic masses \\
\hline modmass & Modification on default atomic masses \\
\hline
\end{tabular}

By default, atomic masses employed in the calculation are loaded from output file of quantum chemistry program, user can also use the "defmass" parameter to request Shermo to employ average atomic masses or masses of principal isotopes. Atomic masses for specific atoms can be further modified by the "modmass" parameter.

Using Shermo to scan temperature and pressure is straightforward, users simply need to specify lower limit, upper limit and stepsize. For example, specifying "T=50,200,10" in the "settings.ini" file or adding "-T 50,200,10" argument in running command will request Shermo to output various thermodynamic data every $10 \mathrm{~K}$ from 50 to $200 \mathrm{~K}$. Two-dimension scan for both temperature and pressure is also supported.

\section{Examples}

In this section, we present two simple examples to briefly illustrate use and practical value of the Shermo program. Shermo 2.0, Gaussian 16. A.03 $3^{[1]}$ and ORCA $4.2 .1^{[2]}$ were utilized in the examples. Molecular structure was plotted by ChimeraX program. ${ }^{[23]}$

\subsection{Formaldehyde}

This example studies thermodynamic data of formaldehyde. Optimization and harmonic frequency analysis were finished at the popular B3LYP/6-31G* level, ${ }^{[24,25]}$ after that electronic energy was evaluated at the significantly more accurate $\operatorname{CSSD}(\mathrm{T}) / \mathrm{cc}-\mathrm{pVQZ}$ level, ${ }^{[26,}{ }^{27]}$ the result was found to be -114.368896 a.u. In order to calculate thermodynamic quantities for the formaldehyde at $350 \mathrm{~K}$, one can simply run this command:

$$
\text { Shermo H2CO_optfreq.out -SClZPE } 0.9806 \text {-T } 350 \text {-E }-114.368896
$$

where 0.9806 is the frequency scale factor fitted for ZPE at B3LYP/6-31G* level.. ${ }^{[17]}$ Ref. [17] also presented scale factor for entropy and heating contribution to enthalpy (1.0015 and 0.9989, respectively), they are not explicitly specified here because they are quite close to the default value (1.0). Note that the default pressure is $1 \mathrm{~atm}$ and the default calculation model is RRHO.

After running the above command, detailed molecular information will be printed on screen, 


$\begin{array}{lllllll}4 & 1850.19 & 2.59366 & 0.00263 & 2.59630 & 0.05726 & 0.00851 \\ 5 & 2914.57 & 4.08576 & 0.00005 & 4.08581 & 0.00179 & 0.00016 \\ 6 & 2965.59 & 4.15727 & 0.00004 & 4.15732 & 0.00150 & 0.00013\end{array}$

From the data a tendency can be observed, that is the lower the frequency of a mode, the larger its contribution to $U(T)-U(0), C_{\mathrm{V}}$ and $S$. In contrast, the higher the frequency of a mode, the larger its contribution to ZPE and $U(T)$.

In order to study variation of various thermodynamic quantities with respect to change of temperature, for example, from 50 to $1000 \mathrm{~K}$ with stepsize of $5 \mathrm{~K}$, one can add "-T 50,1000,10" to the running command. After calculation, two files "scan_SCq.txt" and "scan_UHG.txt" will be generated in current folder, which contain thermodynamic data at various temperatures and can be easily plotted via data visualization softwares such as gnuplot ${ }^{[28]}$ and Origin programs. ${ }^{[29]}$ Fig. 2 exhibits variation of thermal corrections to internal energy, enthalpy and Gibbs free energy with respect to temperature.

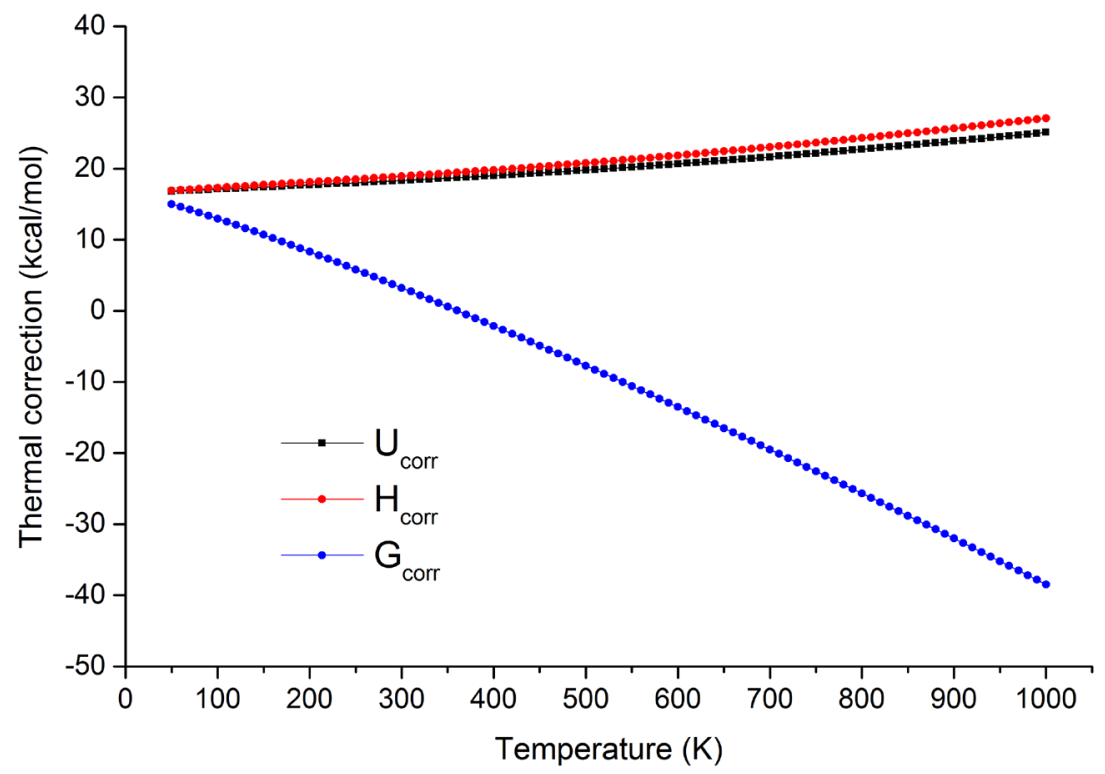

Figure 2 Variation of thermal corrections to internal energy $\left(U_{\text {corr }}\right)$, enthalpy $\left(H_{\text {corr }}\right)$ and Gibbs free energy $\left(G_{\text {corr }}\right)$ with respect to temperature for formaldehyde.

\subsection{Remdesivir}

Remdesivir is a broad-spectrum antiviral medication and recently shown to be the most promising treatment for COVID-19 virus. ${ }^{[30,31]}$ Our recent conformation search study of remdesivir by our Molclus program ${ }^{[32]}$ determined a batch of its low-energy conformations in aqueous environment. ${ }^{[33]}$ The structure of the most stable conformer of the remdesivir is shown in Fig. 3. 


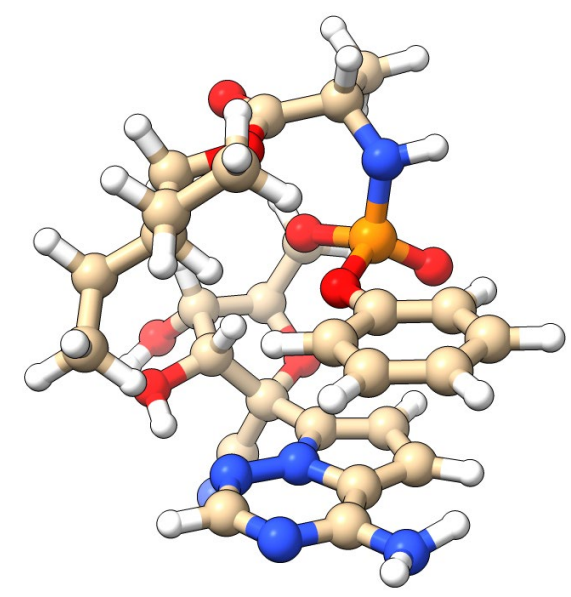

Figure 3 Structure of the most stable conformation of remdesivir in water.

The geometry optimization and harmonic frequency analysis of remdesivir were conducted at B3LYP-D3(BJ)/6-31G* level ${ }^{[24,25,34]}$ by Gaussian. Since remdesivir is a highly flexible molecule with huge number of rotatable bonds, there are as many as 15 and 21 vibrational modes with frequencies lower than 100 and $150 \mathrm{~cm}^{-1}$, respectively. As mentioned earlier, the common RRHO treatment is incapable of reasonably representing contributions to thermodynamic quantities from very low frequency modes like these. In this example, several thermodynamic data were computed at standard condition for remdesivir using RRHO and the two QRRHO models supported by Shermo, the results are summarized in Table 2. To activate the QRRHO models, users only need to add "-ilowfreq 1" or "-ilowfreq 2" argument to command-line or make corresponding modification on the "settings.ini" file.

Table 2 Several thermodynamic data of remdesivir calculated by various models of representing vibrational contribution

\begin{tabular}{cccc}
\hline Model & $U(T)-U(0)(\mathrm{kcal} / \mathrm{mol})$ & $-T S(\mathrm{kcal} / \mathrm{mol})$ & $C_{\mathrm{V}}(\mathrm{cal} / \mathrm{mol} / \mathrm{K})$ \\
\hline RRHO & 24.1 & 49.1 & 151.3 \\
$\mathrm{QRRHO}(1)^{a}$ & 23.2 & 42.4 & 150.9 \\
$\left.\mathrm{QRRHO}^{a}\right)^{b}$ & 24.1 & 43.4 & 151.3 \\
\hline
\end{tabular}

${ }^{a}$ Raising all vibrational frequencies lower than $100 \mathrm{~cm}^{-1}$ to $100 \mathrm{~cm}^{-1}$

${ }^{b}$ Grimme's entropy interpolation between harmonic and free-rotor approximations. Note that this treatment does not alter $U(T)-U(0)$ and $C_{\mathrm{V}}$ of RRHO.

From Table 2 it can be seen that the $U(T)-U(0)$ and $C_{\mathrm{V}}$ calculated under RRHO and QRRHO(1) model do not differ with each other markedly, however, both the two QRRHO models changed the -TS term of RRHO by more than $10 \%$, rendering the importance of applying QRRHO model when calculating Gibbs free energy for very flexible systems like remdesivir. Since the two QRRHO models gave similar results, both of them could be regarded as reasonable and can thus be safely used in practice.

Since flexible systems usually have multiple thermal accessible conformations, the effect of conformation weighting should be properly taken into account in the calculation of their thermodynamic data. Gibbs free energies for a batch of low-energy conformers of remdesivir were previously determined by us using thermal correction at B3LYP-D3(BJ)/6-31G* level and electronic energies evaluated using the robust double-hybrid functional PWPB95-D3(BJ $)^{[35]}$ in combination with high quality def2-QZVPP basis set. ${ }^{[36]}$ For simplicity, in the calculation of conformation weighted 
thermochemistry quantities in this example, only the three conformers with lowest Gibbs free energies are taken into account. After manually creating a conformation list file as mentioned in Section 3.2 and taking it as input file to run Shermo, the thermodynamic quantities and Boltzmann weights of all conformers, as well as conformation weighted data are immediately printed out, as shown below

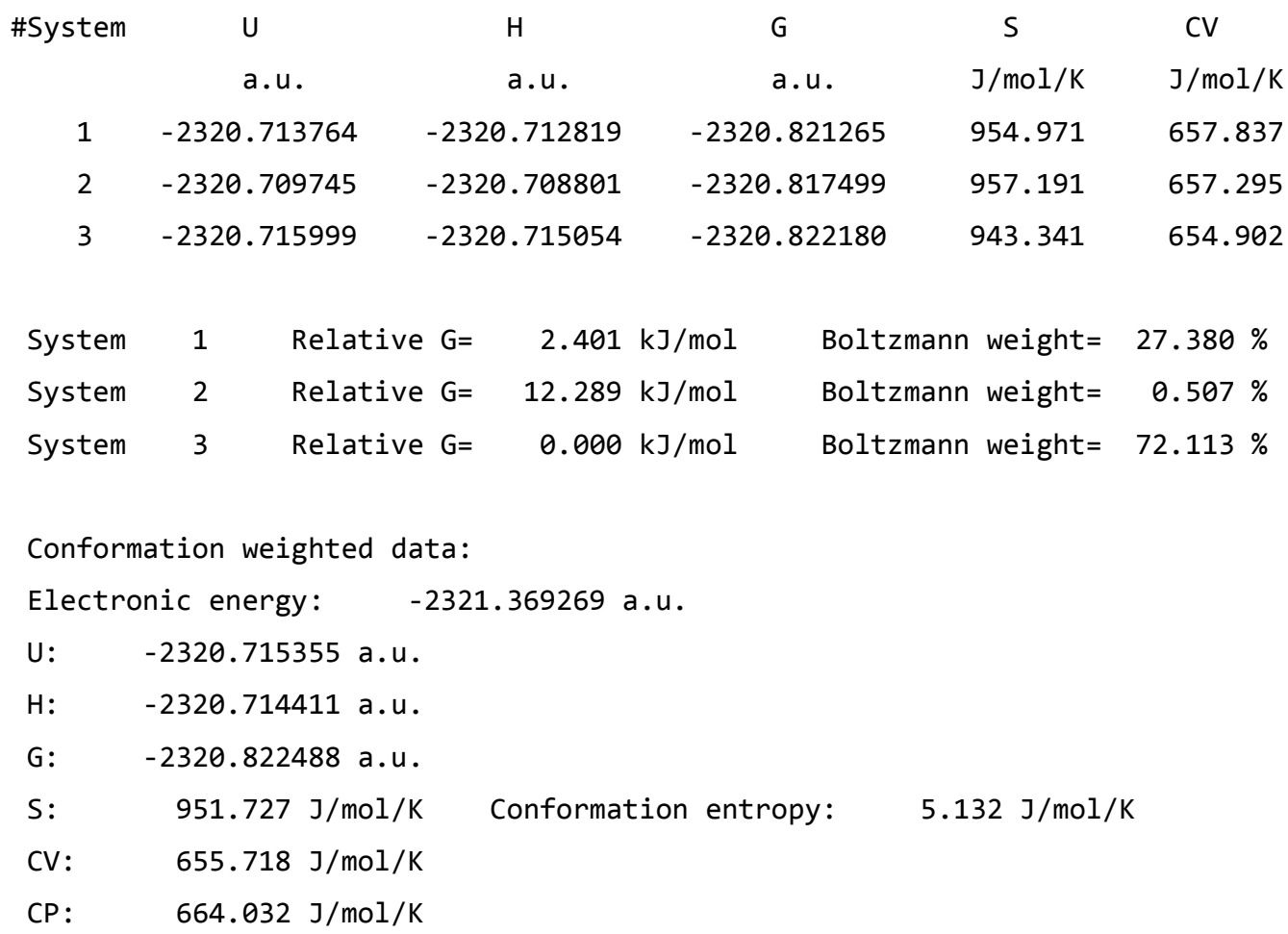

From the data it can be seen that the conformer 3 plays a dominant role, however, the conformer 1 can never be ignored, since its population (27.38\%) is also prominent. The conformer 2 in fact can be ignored, because its Boltzmann weight is close to zero at the current condition $(298.15 \mathrm{~K}$ and 1 atm) due to its relatively high free energy.

\section{Summary}

In this article, we introduced our newly developed code named Shermo, which is designed as a general tool for calculating common molecular thermodynamic quantities. In addition, the theoretical backgrounds involved in Shermo are briefly described, and practical application examples are given. This very user-friendly program makes thermochemistry analysis at different temperatures and pressures as well as with various frequency scale factors much more convenient than ever. Shermo also brings many additional advantages, for example, blackbox QRRHO model can be easily applied to low frequencies, conformation or configuration weighted quantities can be directly obtained, various components of thermodynamic quantities can be studied individually. Due to its usefulness, it is expected that the Shermo program will become a popular code for thermodynamic analysis of molecular systems.

Finally, we would like to mention that although using Shermo in combination with common quantum chemistry programs is able to reasonably study thermodynamic quantities for most majority of molecular systems, if there are very strict requirements on the accuracy of the data, sometimes more advanced models may be needed to further improve representation of the system, which are beyond current scope of the Shermo program. For example, anharmonic effect of certain 
vibrational modes may need to be explicitly represented, ${ }^{[37]}$ torsional motions of some groups or fragments should be represented as hindered rotors, ${ }^{[38]}$ strongly coupled torsions may be better represented via multistructural torsional method. ${ }^{[39]}$

\section{References}

1. M. J. Frisch; G. W. Trucks; H. B. Schlegel; G. E. Scuseria; M. A. Robb; J. R. Cheeseman; G. Scalmani; V. Barone; G. A. Petersson; H. Nakatsuji; X. Li; M. Caricato; A. V. Marenich; J. Bloino; B. G. Janesko; R. Gomperts; B. Mennucci; H. P. Hratchian; J. V. Ortiz; A. F. Izmaylov; J. L. Sonnenberg; Williams; F. Ding; F. Lipparini; F. Egidi; J. Goings; B. Peng; A. Petrone; T. Henderson; D. Ranasinghe; V. G. Zakrzewski; J. Gao; N. Rega; G. Zheng; W. Liang; M. Hada; M. Ehara; K. Toyota; R. Fukuda; J. Hasegawa; M. Ishida; T. Nakajima; Y. Honda; O. Kitao; H. Nakai; T. Vreven; K. Throssell; J. A. Montgomery Jr.; J. E. Peralta; F. Ogliaro; M. J. Bearpark; J. J. Heyd; E. N. Brothers; K. N. Kudin; V. N. Staroverov; T. A. Keith; R. Kobayashi; J. Normand; K. Raghavachari; A. P. Rendell; J. C. Burant; S. S. Iyengar; J. Tomasi; M. Cossi; J. M. Millam; M. Klene; C. Adamo; R. Cammi; J. W. Ochterski; R. L. Martin; K. Morokuma; O. Farkas; J. B. Foresman; D. J. Fox. Gaussian 16 A.03, Wallingford, CT, 2016.

2. F. Neese. Software update: the ORCA program system, version 4.0. WIREs Comput. Mol. Sci., 8, e1327 (2018). DOI: 10.1002/wcms.1327.

3. M. W. Schmidt; K. K. Baldridge; J. A. Boatz; S. T. Elbert; M. S. Gordon; J. H. Jensen; S. Koseki; N. Matsunaga; K. A. Nguyen; S. Su; T. L. Windus; M. Dupuis; J. A. Montgomery. General atomic and molecular electronic structure system. J. Comput. Chem., 14, 1347 (1993). DOI: 10.1002/jcc.540141112. 4. H. J. J. van Dam; W. A. de Jong; E. Bylaska; N. Govind; K. Kowalski; T. P. Straatsma; M. Valiev. NWChem: scalable parallel computational chemistry. WIREs: Comp. Mol. Sci., 1, 888 (2011). DOI: 10.1002/wcms. 62 .

5. K. K. Irikura. Appendix B: Essential Statistical Thermodynamics. In Computational Thermochemistry: Prediction and Estimation of Molecular Thermodynamics (ACS Symposium Series 677), American Chemical Society: Washington, DC, 1998.

6. F. Jensen. Introduction to Computational Chemistry. $3^{\text {rd }}$ ed.; John Wiley \& Sons: West Sussex, 2017; pp 454-459.

7. L. Gyevi-Nagy; G. Tasi. SYVA: A program to analyze symmetry of molecules based on vector algebra. Comput. Phys. Commun., 215, 156 (2017). DOI: 10.1016/j.cpc.2017.01.019.

8. I. M. Alecu; J. Zheng; Y. Zhao; D. G. Truhlar. Computational Thermochemistry: Scale Factor Databases and Scale Factors for Vibrational Frequencies Obtained from Electronic Model Chemistries. J. Chem. Theory Comput., 6, 2872 (2010). DOI: 10.1021/ct100326h.

9. Y. Tantirungrotechai; K. Phanasant; S. Roddecha; P. Surawatanawong; V. Sutthikhum; J. Limtrakul. Scaling factors for vibrational frequencies and zero-point vibrational energies of some recently developed exchange-correlation functionals. J. Mol. Struct. (THEOCHEM), 760, 189 (2006). DOI: 10.1016/j.theochem.2005.12.007.

10. M. K. Kesharwani; B. Brauer; J. M. L. Martin. Frequency and Zero-Point Vibrational Energy Scale Factors for Double-Hybrid Density Functionals (and Other Selected Methods): Can Anharmonic Force Fields Be Avoided? J. Phys. Chem. A, 119, 1701 (2015). DOI: 10.1021/jp508422u.

11. M. P. Andersson; P. Uvdal. New Scale Factors for Harmonic Vibrational Frequencies Using the B3LYP Density Functional Method with the Triple- $\zeta$ Basis Set 6-311+G(d,p). J. Phys. Chem. A, 109, 2937 (2005). DOI: 10.1021/jp045733a. 
12. J. A. Pople; A. P. Scott; M. W. Wong; L. Radom. Scaling Factors for Obtaining Fundamental Vibrational Frequencies and Zero-Point Energies from HF/6-31G* and MP2/6-31G* Harmonic Frequencies. Isr. J. Chem., 33, 345 (1993). DOI: 10.1002/ijch.199300041.

13. R. S. Grev; C. L. Janssen; H. F. Schaefer. Concerning zero - point vibrational energy corrections to electronic energies. J. Chem. Phys., 95, 5128 (1991). DOI: 10.1063/1.461680.

14. B. Chan; L. Radom. Frequency Scale Factors for Some Double-Hybrid Density Functional Theory Procedures: Accurate Thermochemical Components for High-Level Composite Protocols. J. Chem. Theory Comput., 12, 3774 (2016). DOI: 10.1021/acs.jctc.6b00554.

15. J. P. Merrick; D. Moran; L. Radom. An Evaluation of Harmonic Vibrational Frequency Scale Factors. J. Phys. Chem. A, 111, 11683 (2007). DOI: 10.1021/jp073974n.

16. P. Sinha; S. E. Boesch; C. Gu; R. A. Wheeler; A. K. Wilson. Harmonic Vibrational Frequencies: Scaling Factors for HF, B3LYP, and MP2 Methods in Combination with Correlation Consistent Basis Sets. J. Phys. Chem. A, 108, 9213 (2004). DOI: 10.1021/jp048233q.

17. A. P. Scott; L. Radom. Harmonic Vibrational Frequencies: An Evaluation of Hartree-Fock, Møller-Plesset, Quadratic Configuration Interaction, Density Functional Theory, and Semiempirical Scale Factors. J. Phys. Chem., 100, 16502 (1996). DOI: 10.1021/jp960976r.

18. R. F. Ribeiro; A. V. Marenich; C. J. Cramer; D. G. Truhlar. Use of Solution-Phase Vibrational Frequencies in Continuum Models for the Free Energy of Solvation. J. Phys. Chem. B, 115, 14556 (2011). DOI: $10.1021 / \mathrm{jp} 205508 \mathrm{z}$.

19. A. V. Marenich; W. Ding; C. J. Cramer; D. G. Truhlar. Resolution of a Challenge for Solvation Modeling: Calculation of Dicarboxylic Acid Dissociation Constants Using Mixed Discrete-Continuum Solvation Models. J. Phys. Chem. Lett., 3, 1437 (2012). DOI: 10.1021/jz300416r.

20. L. Xu; M. L. Coote. Methods To Improve the Calculations of Solvation Model Density Solvation Free Energies and Associated Aqueous pKa Values: Comparison between Choosing an Optimal Theoretical Level, Solute Cavity Scaling, and Using Explicit Solvent Molecules. J. Phys. Chem. A, 123, 7430 (2019). DOI: 10.1021/acs.jpca.9b04920.

21. S. Grimme. Supramolecular Binding Thermodynamics by Dispersion-Corrected Density Functional Theory. Chem.-Eur. J., 18, 9955 (2012). DOI: 10.1002/chem.201200497.

22. T. Lu; S. Manzetti. Wavefunction and reactivity study of benzo[a]pyrene diol epoxide and its enantiomeric forms. Struct. Chem., 25, 1521 (2014). DOI: 10.1007/s11224-014-0430-6.

23. T. D. Goddard; C. C. Huang; E. C. Meng; E. F. Pettersen; G. S. Couch; J. H. Morris; T. E. Ferrin. UCSF ChimeraX: Meeting modern challenges in visualization and analysis. Protein Sci., 27, 14 (2018). DOI: $10.1002 /$ pro.3235.

24. P. J. Stephens; F. J. Devlin; C. F. Chabalowski; M. J. Frisch. Ab Initio Calculation of Vibrational Absorption and Circular Dichroism Spectra Using Density Functional Force Fields. J. Phys. Chem., 98, 11623 (1994). DOI: 10.1021/j100096a001.

25. P. C. Hariharan; J. A. Pople. The influence of polarization functions on molecular orbital hydrogenation energies. Theor. Chem. Acc., 28, 213 (1973).

26. J. T. H. Dunning. Gaussian Basis Sets for Use in Correlated Molecular Calculations. I. The Atoms Boron through Neon and Hydrogen. J. Chem. Phys., 90, 1007 (1989).

27. A. Szabo; N. S. Ostlund. Modern Quantum Chemistry. Dover Publications: New York, 1989.

28. gnuplot program, http://www.gnuplot.info (accessed on May 15, 2020).

29. Origin program, https://www.originlab.com (accessed on May 15, 2020).

30. Remdesivir, https://en.wikipedia.org/wiki/Remdesivir (accessed on May 15, 2020). 
31. C. Scavone; S. Brusco; M. Bertini; L. Sportiello; C. Rafaniello; A. Zoccoli; L. Berrino; G. Racagni; F. Rossi; A. Capuano. Current pharmacological treatments for COVID-19: what's next? Br. J. Pharmacol. (2020). DOI: $10.1111 / \mathrm{bph} .15072$.

32. Tian Lu, molclus program, http://www.keinsci.com/research/molclus.html (accessed on May 15, 2020).

33. Tian Lu, Conformation search for remdesivir using Molclus program in combination with molecular dynamics simulation by xtb program, http://bbs.keinsci.com/thread-16255-1-1.html (accessed on May $15,2020)$.

34. S. Grimme; J. Antony; S. Ehrlich; H. Krieg. A consistent and accurate ab initio parametrization of density functional dispersion correction (DFT-D) for the 94 elements H-Pu. J. Chem. Phys., 132, 154104 (2010).

35. L. Goerigk; S. Grimme. Efficient and Accurate Double-Hybrid-Meta-GGA Density FunctionalsEvaluation with the Extended GMTKN30 Database for General Main Group Thermochemistry, Kinetics, and Noncovalent Interactions. J. Chem. Theory Comput., 7, 291 (2011). DOI: 10.1021/ct100466k.

36. F. Weigend; R. Ahlrichs. Balanced basis sets of split valence, triple zeta valence and quadruple zeta valence quality for $\mathrm{H}$ to Rn: Design and assessment of accuracy. Phys. Chem. Chem. Phys., 7, 3297 (2005). DOI: 10.1039/b508541a.

37. V. Barone. Anharmonic vibrational properties by a fully automated second-order perturbative approach, 122, 014108 (2005). DOI: 10.1063/1.1824881.

38. P. Y. Ayala; H. B. Schlegel. Identification and treatment of internal rotation in normal mode vibrational analysis. J. Chem. Phys., 108, 2314 (1998). DOI: 10.1063/1.475616.

39. D. Ferro-Costas; M. N. D. S. Cordeiro; D. G. Truhlar; A. Fernández-Ramos. Q2DTor: A program to treat torsional anharmonicity through coupled pair torsions in flexible molecules. Comput. Phys. Commun., 232, 190 (2018). DOI: https://doi.org/10.1016/j.cpc.2018.05.025. 